\title{
Effect of Lipid Composition and Preparation Method on Properties of Ferulic Acid Encapsulated Liposomes
}

\author{
K.M. Geethi K. Pamunuwa, Veranja Karunaratne and D. Nedra Karunaratne
}

\begin{abstract}
In this study, the effect of charge which is brought about by the lipid composition and method of preparation on the properties of ferulic acid encapsulated liposomes was investigated to determine the optimum conditions with respect to mainly in vitro release and ex vivo skin permeation of ferulic acid. Positively charged liposomes exhibited higher encapsulation efficiencies than negatively charged liposomes. However, positively charged liposomes were much larger and less homogenous than negatively charged liposomes, which may influence many properties including biodistribution. It was revealed that positively charged liposomes are better for slow release while negatively charged liposomes are better for skin permeation properties.
\end{abstract}

Keywords - liposomal encapsulation, ferulic acid, slow release, skin permeation

\section{Introduction}

Liposomes, vesicular structures with lipid bilayers and an aqueous interior, play a pivotal role as drug delivery vehicles, in pharmaceutical industry and in cosmetic industry. Therefore, numerous strategies are adopted to prepare liposomes with varying properties, thus enabling those liposomes to function even more effectively [1].

The model bioactive agent used in this study is ferulic acid which is a potent antioxidant having numerous other important bioactivities [2,3]. It is an amphiphilic molecule which shows intermediate solubility in water, and thus this drug is expected to behave differently than both hydrophilic and hydrophobic encapsulants. Liposomal ferulic acid has been the subject of investigations of numerous research groups [4,5]. However, the effect of method of preparation and charge on properties of ferulic acid encapsulated liposomes has remained unexplored.

K.M. Geethi K. Pamunuwa, Veranja Karunaratne, D. Nedra Karunaratne Department of Chemistry, Faculty of Science, University of Peradeniya Sri Lanka

K.M. Geethi K. Pamunuwa

Postgraduate Institute of Science, University of Peradeniya

Sri Lanka

Veranja Karunaratne

Sri Lanka Institute of Nanotechnology

Sri Lanka
Changing the method of preparation of liposomes usually alters properties such as size, lamellarity, and encapsulation efficiency; and hence, may affect release kinetics and skin permeation properties [6]. Thus, ferulic acid encapsulated liposomes were prepared using three methods - reverse phase evaporation method, thin film hydration method and proliposome method - and the properties of those liposomes were evaluated and compared.

Changing the lipid composition, also, alters the properties of liposomes. In fact numerous authors have reported the effect of charge, which is brought about by changing the lipid composition of liposomes, on the properties of liposomes $[7,8]$. Thus, both negatively charged liposomes and positively charged liposomes, encapsulating ferulic acid were prepared, and the properties of those liposomes were evaluated and compared.

The aim of this research project was to investigate the effect of method of preparation and lipid composition on the properties of ferulic acid encapsulated liposomes. Furthermore, the effect of liposomal encapsulation on release properties and skin permeation properties of ferulic acid was evaluated. In addition to providing insight into the optimum method/s and lipid compositions for the preparation of ferulic acid encapsulated liposomes, this study will provide knowledge that will be very useful for future engineering of liposomal formulations encapsulating amphiphilic drugs and bioactive agents.

\section{Materials and methods}

\section{A. Materials}

Egg yolk phosphatidylcholine (PC) ( 60\% TLC), cholesterol $(\mathrm{CH})$ (assay > 98\%), stearylamine (SA) (assay 90 $\%$ ) and ferulic acid ( $\geq 99.0 \%$, HPLC) were purchased from Sigma-Aldrich. Dichloromethane, ethanol and methanol were from Sigma. Other chemicals were of analytical grade. Dialysis tubing (12 000 MWCO) was from Sigma-Aldrich. Fresh pig ears were obtained from a local slaughter house. Deionized water filtered through a $0.2 \mu \mathrm{m}$ filter was used for all experiments. 


\section{B. Methods}

- $\quad$ Preparation of ferulic acid encapsulated liposomes

Both negatively charged ferulic acid encapsulated liposomes $(\mathrm{N})$ and positively charged ferulic acid encapsulated liposomes $(\mathrm{P})$ were prepared by the reverse phase evaporation method (REV), thin-film hydration method (TFH) and proliposome method (PRO). Phosphate buffered saline (PBS; $\mathrm{pH}$ 7.4) was used as the aqueous medium. The amounts of chemicals used in the preparation of liposomes are given in Table 1.

- Determination of encapsulation efficiency (EE) and loading capacity (LC)

EE and LC of ferulic acid encapsulated liposomes were determined using a spectrophotometric method. Ferulic acid was quantified by measuring absorbance at $321 \mathrm{~nm}$. The formula used for the calculation of EE and LC are given below.

$$
\mathrm{EE}=\frac{\begin{array}{c}
\text { Total amount } \\
\text { of } \\
\text { ferulic acid }
\end{array}}{-\begin{array}{c}
\text { Amount of } \\
\text { ferulic acid in } \\
\text { supernatant }
\end{array}} \times 100
$$

$$
\begin{aligned}
& \text { Mass of encapsulated ferulic acid } \\
& \mathrm{LC}=\frac{\text { Mass of ferulic acid encapsulated }}{\text { liposomes }} \times 100
\end{aligned}
$$

- Determination of particle size and zeta-potential

Particle sizes and zeta-potentials of liposomes were determined using a Malvern zetasizer NanoZS (Malvern instruments, UK) fitted with a red laser of $633 \mathrm{~nm}$, using dynamic light scattering technique and laser doppler electrophoresis technique, respectively, after equilibrating the samples at $25{ }^{\circ} \mathrm{C}$. The values reported are the z-average diameters and zeta-potentials of liposomes.

\section{- In vitro release studies}

In vitro release studies were conducted using free ferulic acid and ferulic acid encapsulated liposomes following the dialysis bag method using PBS as the release medium. The withdrawn aliquots from the release medium at predetermined time intervals were used for the determination of released ferulic acid.

\section{- Ex vivo skin permeation studies}

Skin permeation experiments were carried out using a Franz-diffusion cell using excised full thickness pig ear skin as the model membrane. PBS was used as the receiver medium. Ex vivo skin permeation experiments were carried out using free ferulic acid and ferulic acid encapsulated liposomes.

Average steady state flux (J) was calculated using the following equation.

$$
J=(d Q / d t) / A
$$

where: A - Surface area of the skin, and dQ/dt - Slope of the plot Q vs. t

The skin permeability $\left(\mathrm{K}_{\mathrm{p}}\right)$ is given by the following equation.

$$
\mathrm{K}_{\mathrm{p}}=\mathrm{J} / \Delta \mathrm{C}
$$

where, $\Delta \mathrm{C}-$ Difference in drug concentration of donor and receiver compartments at a given time

The average permeability ( $\mathrm{K}_{\mathrm{p}}(\mathrm{ave})$ ) was calculated using the following equation.

$$
\mathrm{K}_{\mathrm{p}}(\mathrm{ave})=\left\{\sum(\mathrm{J} / \Delta \mathrm{C})\right\} / \mathrm{N}
$$

where, $\mathrm{N}$ - Number of intervals

\section{- $\quad$ Statistical analysis}

All data are presented as mean \pm standard deviation (S.D.) of three parallel experiments $(\mathrm{n}=3)$. Microsoft Office Excel 2007 was used for the above calculations. One way ANOVA was conducted using MINITAB 14 software to compare the results and $\mathrm{P}<0.05$ was considered significant.

\section{Results and discussion}

In this study, the effect of charge and method of preparation of ferulic acid encapsulated liposomes was evaluated not only on the basic physical properties but also on

TABLE 1. THE AMOUNTS OF CHEMICALS USED IN THE PREPARATION OF FERULIC ACID ENCAPSULATED LIPOSOMES

\begin{tabular}{|c|c|c|c|c|}
\hline Liposomal formulation & $\begin{array}{c}\text { PC } \\
(\mathbf{m g})\end{array}$ & $\begin{array}{c}\text { CH } \\
(\mathbf{m g})\end{array}$ & $\begin{array}{c}\text { Ferulic acid } \\
(\mathbf{m g})\end{array}$ & - \\
\hline N-REV & 100 & 40 & 40 & - \\
\hline N-TFH & 100 & 40 & -0 \\
\hline N-PRO & 100 & 40 & \\
\hline P-REV & 100 & 1.0 & \\
\hline P-TFH & 100 & 40 & & \\
\hline P-PRO & 100 & 40 & 1.0 & 20 \\
\hline
\end{tabular}


other pharmaceutically and cosmeceutically important properties such as in vitro release and skin permeation properties.

\section{A. Encapsulation efficiency and loading capacity}

EEs and LCs of different liposomal formulations are shown in Table 2. According to our results, the charge of liposomes has a significant effect on EE of ferulic acid encapsulated liposomes. In fact, the EEs of the two types of positively charged liposomes were significantly higher than those of the three types of negatively charged liposomes. SA, which is the chemical compound incorporated in the lipid bilayer to impart a positive charge to liposomes, remains protonated at neutral $\mathrm{pH}$, and thus, may interact strongly with deprotonated negatively charged ferulic acid molecules via mainly electrostatic interactions. These interactions may be the cause of the higher EEs exhibited by positively charged liposomes. Thus, positively charged liposomes, especially those vesicles containing SA, may be more appropriate for the encapsulation of ferulic acid with high encapsulation efficiencies.

The LCs of ferulic acid encapsulated liposomes approximated $0.5 \%$. This relatively low value may be a result of the low drug/lipid ratio used in the preparation of liposomes. This study indicates that the lipid compositions and methods utilized in this study are equally appropriate to obtain ferulic acid encapsulated liposomes with a LC of $0.5 \%$.

\section{B. Particle size and zeta-potential}

Particle sizes and zeta-potentials of ferulic acid encapsulated liposomes were analyzed and the values are given in Table 2. As indicated in Table 2, the incorporation of $\mathrm{SA}$ in the lipid bilayer/s of ferulic acid encapsulated liposomes increases the size of those vesicles. Moreover, the method of preparation has a significant effect on the size of ferulic acid encapsulated liposomes. For instance, the two types of positively charged liposomes are different in size, such that the average diameter of P-PRO is much larger than that of P-TFH. Therefore, thin film hydration method may be utilized to prepare smaller (i.e. approximately $400 \mathrm{~nm}$ ) positively charged ferulic acid encapsulated liposomes.

Polydispersity index is indicative of the homogeneity of the liposomes in size, which is of utmost importance in most instances for improved therapeutic efficacy of liposomal drugs. As indicated in Table 2, the incorporation of SA in

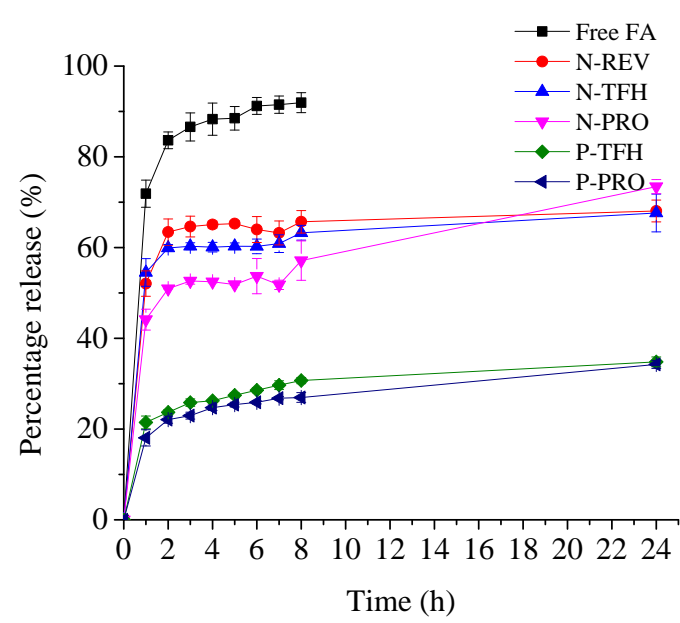

FIGURE 1. IN VITRO RELEASE PROFILES OF FREE FERULIC ACID AND DIFFERENT LIPOSOMAL FORMULATIONS

liposomes results in liposomes less homogenous in size. However, the polydispersity indeces of positively charged liposomes are dependent on the method of preparation. Our results show that thin film hydration method may be used to yield positively charged liposomes with high homogeneity.

The zeta-potential which is indicative of the charge is a significant parameter of liposomes. According to Table 2, the zeta-potential of ferulic acid encapsulated liposomes depend heavily on the lipid composition. Specifically, the incorporation of SA in the lipid bilayers of liposomes made of $\mathrm{PC}$ and $\mathrm{CH}$ results in positively charged liposomes.

\section{In vitro release studies}

In vitro release properties of free ferulic acid and ferulic acid encapsulated liposomes that differ in lipid composition, charge and type were evaluated and the release profiles are depicted in Fig. 1.

The five types of ferulic acid encapsulated liposomes exhibited much slower release of ferulic acid than free ferulic acid. Moreover, release kinetics conformed to the Gompertz model which shows a steep increase in the beginning that converged slowly to asymptotic maximal dissolution. Thus, liposomal encapsulation is an effective means of improving slow release of ferulic acid irrespective of the charge or type of liposomes. Moreover, these results show that the release properties may be improved further by utilizing positively

TABLE 2. DIAMETER, POLYDISPERSITY INDEX AND ZETA-POTENTIAL OF DIFFERENT LIPOSOMAL FORMULATIONS. EACH VALUE REPRESENTS MEAN \pm S.D. $(\mathrm{N}=3)$. SIGNIFICANTLY DIFFERENT VALUES ARE FOLLOWED BY DIFFERENT SUPERSCRIPTS AND VICE VERSA $(\mathrm{P}<0.05)$

\begin{tabular}{|c|c|c|c|}
\hline Liposomal formulation & Diameter (nm) & Polydispersity index & Zeta-potential (mV) \\
\hline N-REV & $289.5 \pm 9.7^{\mathrm{a}}$ & $0.242 \pm 0.014^{\mathrm{a}}$ & $-63.6 \pm 3.4^{\mathrm{a}}$ \\
\hline N-TFH & $329.8 \pm 7.0^{b}$ & $0.206 \pm 0.002^{\mathrm{a}}$ & $-61.6 \pm 4.5^{\mathrm{a}}$ \\
\hline N-PRO & $253.5 \pm 1.8^{\mathrm{a}}$ & $0.254 \pm 0.011^{\mathrm{a}}$ & $-61.3 \pm 3.4^{\mathrm{a}}$ \\
\hline P-REV & Not determined & Not determined & Not determined \\
\hline P-TFH & $420.3 \pm 27.0^{c}$ & $0.263 \pm 0.023^{\mathrm{a}}$ & $+20.1 \pm 5.9^{b}$ \\
\hline P-PRO & $624.4 \pm 17.2^{\mathrm{d}}$ & $0.389 \pm 0.053^{b}$ & $+6.3 \pm 2.1^{\mathrm{c}}$ \\
\hline
\end{tabular}




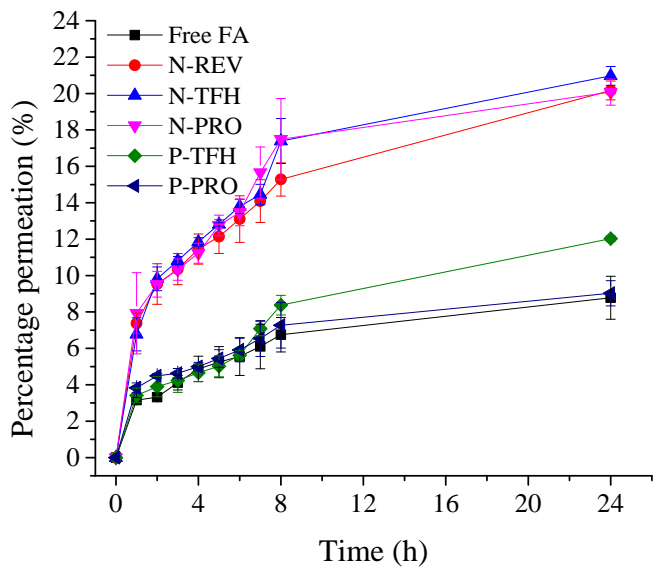

FIGURE 1. EX VIVO SKIN PERMEATION PROFILES OF FREE FERULIC ACID AND DIFFERENT LIPOSOMAL FORMULATIONS

charged ferulic acid encapsulated liposomes.

Specifically, incorporating SA in the lipid bilayers of ferulic acid encapsulated liposomes is an effective strategy that enhances slow release of ferulic acid. One major reason for the slower release properties exhibited by SA-containing liposomes may the strong electrostatic interactions of SA and ferulic acid, that hinder the escape of ferulic acid from liposomes.

\section{Ex vivo skin permeation studies}

The skin permeation profiles of free ferulic acid and different liposomal formulations are depicted in Fig. 2 and values pertaining to skin permeation experiments are shown in Table 3.

As depicted in Fig. 2, the three types of negatively charged liposomes exhibited much greater skin permeation of ferulic acid than both the two types of positively charged liposomes and free ferulic acid in PBS. In fact, the cumulative percent permeation of ferulic acid at $24 \mathrm{~h}$ of negatively charged liposomes was greater than two-fold than those of free ferulic acid and positively charged liposomes. Thus, the skin permeation of liposomal ferulic acid depends clearly on the charge of liposomes. Also, our results reveal that negatively charged liposomes may be utilized to facilitate skin penetration of ferulic acid.

Although cumulative skin permeation, average flux and average permeability were highly dependent on the charge of liposome, percentage skin deposition and skin deposition per unit area appear to be independent of the charge or method of preparation of ferulic acid encapsulated liposomes (Table 3). Basically, these results indicate that either negatively charged or positively charged liposomes may be utilized for the skin deposition of ferulic acid.

\section{Conclusions}

Charge and/or method of preparation impart significant effects on properties such as EE, LC, size, polydispersity index, zeta-potential, release properties and skin permeation properties of ferulic acid encapsulated liposomes.

Positively charged liposomes prepared by incorporating SA in the lipid bilayers of liposomes may be utilized to obtain liposomes with higher EEs (i.e. approx. $80 \%$ ) than negatively charged liposomes. In general, LC was independent of both charge and method of preparation.

The charge and method of preparation have a profound effect on size of ferulic acid encapsulated liposomes. The polydispersity index also depends on charge, and this parameter indicates that negatively charged liposomes more homogenous than positively charged liposomes, may be prepared under the experimental conditions of this study. The zeta-potential depends on the lipid composition and indicates that egg yolk PC and $\mathrm{CH}$ may be utilized to form stable negatively charged ferulic acid encapsulated liposomes while egg yolk PC, CH and SA may be utilized to form positively charged liposomes.

This study reveals that liposomal encapsulation of ferulic acid, irrespective of the method of preparation, may be utilized for slow release of ferulic acid. As expected, the release of ferulic acid depends on the charge of liposomes. In fact, positively charged liposomes may be employed for much slower release of ferulic acid than negatively charged liposomes.

Also, this work shows that negatively charged liposomes

TABLE 3. CUMULATIVE PER CENT PERMEATION AT $24 \mathrm{H}$, AVERAGE FLUX (J(AVE)), AVERAGE PERMEABILITY (K, (AVE)), SKIN DEPOS IIION PER UNIT AREA AND CUMULATIVE PERCENT SKIN DEPOS IIION AT 24 h FR OM FREE FERULIC ACID AND DIFFERENT FERULIC ACID-CONTAINING LIPOS OMAL FORMULATIONS. EACH VALUE REPRESENTS $M E A N=S D$. (N=3).

\begin{tabular}{|c|c|c|c|c|c|}
\hline $\begin{array}{l}\text { Ferulic acid- } \\
\text { containing } \\
\text { formulation }\end{array}$ & $\begin{array}{c}\text { Cumulative percent } \\
\text { permeation at } 24 \mathrm{~h} \\
(\%)\end{array}$ & $\begin{array}{l}\mathrm{J}(\text { ave }) / 10^{-1} \\
\left(\mu \mathrm{g} / \mathrm{h} \cdot \mathrm{cm}^{2}\right)\end{array}$ & $\begin{array}{c}\mathrm{K}_{p}(\text { ave }) / 10^{-3} \\
(\mathrm{~cm} / \mathrm{h})\end{array}$ & $\begin{array}{l}\text { Slin dep osition } \\
\text { per unit area } \\
\left(\mu \mathrm{g} / \mathrm{cm}^{-2}\right)\end{array}$ & $\begin{array}{l}\text { Cumulative percent shin } \\
\text { deposition at } 24 \mathrm{~h} \\
(\%)\end{array}$ \\
\hline Free FA & $8.8 \pm 1.2^{\mathrm{z}}$ & $2.0 \pm 0.3^{2}$ & $3.2 \pm 0.5^{2}$ & $1.3=0.1$ & $2.3 \pm 0.1^{12}$ \\
\hline N-REV & $20.2 \pm 0.5^{6}$ & $4.1 \pm 0.1^{6}$ & $8.1 \pm 0.2^{6}$ & $1.5 \pm 0.2$ & $3.1 \pm 0.5^{a}$ \\
\hline $\mathrm{N}-\mathrm{TFH}$ & $21.0 \pm 0.5^{b}$ & $4.4 \pm 0.1^{b}$ & $8.4 \pm 0.3^{b}$ & $1.5 \pm 0.1$ & $2.9 \pm 0.2^{a b}$ \\
\hline N-PRO & $20.1 \pm 0.7^{2}$ & $4.3=0.2^{\circ}$ & $8.0=0.3^{\circ}$ & $1.5 \pm 0.2$ & $2.9 \pm 0.3^{27}$ \\
\hline P-TFH & $12.0 \pm 0.2^{e}$ & $2.8 \pm 0.0^{6}$ & $4.5 \pm 0.1^{e}$ & $1.3 \pm 0.3$ & $2.3 \pm 0.4^{27}$ \\
\hline P-PRO & $9.0 \pm 0.7^{\mathrm{a}}$ & $2.1 \pm 0.2^{2}$ & $3.3 \pm 0.3^{2}$ & $1.2 \pm 0.2$ & $2.2 \pm 0.3^{b}$ \\
\hline
\end{tabular}


are superior to positively charged liposomes for skin penetration of ferulic acid. However, both negatively charged liposomes and positively charged liposomes are equally effective in skin deposition of ferulic acid. The method of preparation has only negligible effect on skin permeation and skin deposition of ferulic acid.

\section{Acknowledgment}

The National Science Foundation of Sri Lanka is acknowledged for providing financial assistance through Grant No. NSF/SCH/2013/01.

\section{References}

[1] A.Laouini, C,Jaafar-Maalej, I. Limayem-Blouza, S. Sfar, C. Charcosset and H. Fessi, "Preparation, Characterization and Applications of Liposomes: State of the Art," J Colloid Sci Biotechnol, vol. 1, 2012, pp. $147-168$.

[2] F-H. Lin, J-Y. Lin, R.D. Gupta, J.A. Tournas, J.A. Burch, M.A. Selim, N.A. Monteiro-Riviere, J.M. Grichnik, J. Zielinski and S.R. Pinnell, "Ferulic Acid Stabilizes a Solution of Vitamin C and E and Doubles its Photoprotection of Skin", J Investig Dermotol, vol. 125, 2005, pp. 826832.

[3] T. Ogiwara, K. Satoh, Y. Kadoma, Y. Murakami, S. Unten, T. Atsumi, H. Sakagami and S. Fujisawa, "Radical Scavenging Activity and Cytotoxicity of Ferulic Acid", Anticancer Research. vol. 22(5), 2002, pp. 2711-2717.

[4] M. Chen, X. Liu and A. Fahr, "Skin Delivery of Ferulic Acid from Different Vesicular Systems", Journal of Biomedical Nanotechnology, vol. 6(5), 2010, pp. 577-585.

[5] J. Qin, D.W. Chen, W.G. Lu, H. Xu, C.Y. Yan, H.Y. Hu, B.Y. Chen, M.X. Qiao and X.L. Zhao, "Preparation, Characterization, and Evaluation of Liposomal Ferulic Acid In Vitro and In Vivo", Drug development and industrial pharmacy, vol. 34(6), 2008, pp. 602-608.

[6] A. Akbarzadeh, R. Rezaei-Sadabady, S. Davaran, S.W. Joo, N. Zarghami, Y. Hanifehpour, M. Samiei, M. Kouhi and K. Nejati-Koshki, "Liposome: classification, preparation, and applications. Nanoscale Research Letters", vol. 8, 2013, pp. 102-110.

[7] A. Gillet, P. Compère, F. Lecomte, P. Hubert, E. Ducat, B. Evrard and G. Piel, "Liposome Surface Charge Influence on Skin Penetration Behaviour", International Journal of Pharmaceutics, vol. 411, 2011, pp. 223-231.

[8] S-J. Park, S.G. Choi, E. Davaa and J-S. Park, "Encapsulation Enhancement and Stabilization of Insulin in Cationic Liposomes" International Journal of Pharmaceutics. vol. 415, 2011, pp. 267-272. 